\title{
Tamanho de parcela e número de repetições na cultura do milheto em épocas de avaliação
}

\author{
Cláudia Burin ('); Alberto Cargnelutti Filho (2*); Bruna Mendonça Alves (1); Marcos Toebe (3); \\ Jéssica Andiara Kleinpaul (4); Ismael Mario Márcio Neu $\left({ }^{4}\right)$ \\ (') Universidade Federal de Santa Maria (UFSM), Programa de Pós-graduação em Agronomia, Avenida Roraima, 1.000, \\ 97105-900 Santa Maria (RS), Brasil. \\ (2) UFSM, Departamento de Fitotecnia, 97105-900 Santa Maria (RS), Brasil. \\ (3) Fundação Universidade Federal do Pampa (UNIPAMPA), Rua Luiz Joaquim de Sá Britto, s/n, 97650000 Itaqui (RS), Brasil. \\ (4) UFSM, Curso de Agronomia, 97105-900 Santa Maria (RS), Brasil. \\ (*) Autor correspondente: alberto.cargnelutti.filho@gmail.com
}

Recebido: 27/dez./2014; Aceito: 16/mar./2015

\begin{abstract}
Resumo
Os objetivos deste trabalho foram determinar o tamanho ótimo de parcela (Xo) e o número de repetições para avaliar a massa verde de parte aérea de milheto em épocas de avaliação. Foram conduzidos 42 ensaios de uniformidade de $6 \mathrm{~m} \times 4 \mathrm{~m}$ $\left(24 \mathrm{~m}^{2}\right.$ ), sendo cada ensaio dividido em 24 unidades experimentais básicas (UEB) de $1 \mathrm{~m} \times 1 \mathrm{~m}\left(1 \mathrm{~m}^{2}\right)$. Aos 52,69 e 82 dias após a semeadura, foram avaliados, respectivamente 18, 6 e 18 ensaios, nos quais se pesou a massa verde das plantas de cada UEB. O Xo foi determinado pelo método da curvatura máxima do modelo do coeficiente de variação, e as comparações de médias entre as épocas de avaliação foram realizadas pelo teste t de Student. O número de repetições, para experimentos nos delineamentos inteiramente casualizados e blocos ao acaso, em cenários formados pelas combinações de i tratamentos ( $i=3,4, \ldots, 50)$ e d diferenças mínimas entre médias de tratamentos a serem detectadas como significativas a 5\% de probabilidade, pelo teste de Tukey, expressas em percentagem da média do experimento ( $d=10 \%, 12 \%, \ldots, 30 \%)$, foi realizado por processo iterativo até a convergência. O tamanho ótimo de parcela para avaliar a massa verde de parte aérea de milheto é de 4,46 m², para as três épocas de avaliação. Para avaliar até 50 tratamentos, nos delineamentos inteiramente casualizados e blocos ao acaso, quatro repetições são suficientes para que diferenças entre médias de tratamentos de 28,75\% da média do experimento sejam significativas, pelo teste de Tukey, a 5\% de probabilidade.
\end{abstract}

Palavras-chave: Pennisetum glaucum L., ensaio de uniformidade, planejamento experimental, precisão experimental.

\section{Plot size and number of repetitions in evaluation times in millet crop}

\section{Abstract}

The objectives of this work were to determine the optimum plot size $\left(X_{0}\right)$ and number of repetitions, to evaluate the fresh weight of aerial part of millet, in evaluation times. Forty-two uniformity trials with $6 \mathrm{~m} \times 4 \mathrm{~m}\left(24 \mathrm{~m}^{2}\right)$ were conducted. Each trial was divided in 24 experimental units basic (UEB) with $1 \mathrm{~m} \times 1 \mathrm{~m}\left(1 \mathrm{~m}^{2}\right)$. At 52, 69 and 82 days after sowing were evaluated, respectively, 18, 6 and 18 trials, where the fresh weight of plants was weighed in each UEB. The Xo was determined by the method of maximum curvature of the model coefficient of variation and the means compared, among evaluation times, by Student's $t$ test. The number of repetitions for experiments on completely randomized and randomized block designs, in scenarios of combinations of $i$ treatments $(i=3,4, \ldots, 50)$ and $d$ minimal differences between treatments means, to be detected as significant, $5 \%$ probability by Tukey test, expressed in percentage of the average of the experiment ( $d=10 \%, 12 \%, \ldots, 30 \%)$, was determined by iterative process until convergence. The optimum plot size to evaluate the fresh weight of aerial part of millet is $4.46 \mathrm{~m}^{2}$, to the three evaluation times. To evaluate up to 50 treatments, in completely randomized and randomized block designs, four replications are sufficient for differences between treatment means of $28.75 \%$ of the average experiment are significant at 5\% probability, by Tukey's test.

Key words: Pennisetum glaucum L., uniformity trials, experimental design, experimental precision.

\section{INTRODUÇÃO}

O milheto (Pennisetum glaucum (L.) R. Brown) é uma gramínea anual que apresenta potencial de cobertura de solo e lenta decomposição dos resíduos vegetais
(Boer et al., 2008), destacando-se pela capacidade de reciclagem de nutrientes, principalmente nitrogênio e potássio (Cazetta et al., 2005). A cultura tem ampla 
aplicabilidade, sendo utilizada como forragem de alto valor nutritivo, na produção de grãos, como pastagem e na produção de silagem (Buso et al., 2014).

Como planta de cobertura de solo, o milheto alcançou produção de massa seca de $11,83 \mathrm{t} \mathrm{ha}^{-1}$ e massa verde de 48,54 tha $^{-1}$, em sucessão de culturas (Suzuki \& Alves, 2006). Estudos revelam ainda o alto potencial de produção de massa verde da cultura quando colhida em épocas de avaliação e em diferentes estádios fenológicos, visando diferentes formas da sua utilização (Guimarães et al., 2009; Priesnitz et al., 2011). Em consórcio com leguminosas, o milheto apresentou quantidade de massa verde semelhante ao seu cultivo exclusivo, com produçáo de massa seca de $14,18 \mathrm{tha}^{-1}$ e massa verde de $45,76 \mathrm{tha}^{-1}$, confirmando o alto potencial na cobertura de solo, proporcionando proteçáo ao solo e maior retençấo de umidade, o que favorece os cultivos subsequentes (Oliveira et al., 2002).

A massa verde de plantas é um caractere importante a ser avaliado, pois revela o potencial e o desempenho de plantas de cobertura de solo e seus benefícios para o solo e para as culturas sucessoras. O planejamento de experimentos em culturas de cobertura de solo, principalmente na mensuração de massa verde, por meio de adequado tamanho de parcela e o número de repetiçóes, é importante para minimizar o erro experimental, e, consequentemente, aumentar a precisão experimental e a confiabilidade dos resultados. Dentre as metodologias usadas para a determinação do tamanho ótimo de parcela, o método da curvatura máxima do modelo do coeficiente de variação (Paranaíba et al., 2009) é considerado adequado. Para que seja possível a utilização dessa metodologia, é necessário ter ensaios de uniformidade, a partir dos quais são obtidos dados para estimar o tamanho ótimo de parcela. Esses ensaios não apresentam tratamentos, e os tratos culturais são homogêneos em toda área experimental (Ramalho et al., 2012; Storck et al., 2011). Para a realizaçáo de avaliaçóes de caracteres da cultura em estudo, a área experimental é dividida em unidades experimentais básicas (UEB) de menor dimensão possível.

Estudos com a cultura de milheto, nas diversas áreas em que a espécie é usada, mostram que não há padronização no tamanho de parcela e no número de repetiçóes utilizados nos experimentos. Nesse sentido, Finholdt et al. (2009) utilizaram parcelas experimentais de $5 \mathrm{~m}^{2}(2,5 \mathrm{~m} \times 2 \mathrm{~m})$, com área útil de $4 \mathrm{~m}^{2}$ e quatro repetiçóes, para a avaliação da produçáo de biomassa e cobertura de solo proporcionada pelos principais adubos verdes, entre eles o milheto. Já Priesnitz et al. (2011), estudando a relação do espaçamento entre linhas com o rendimento de biomassa verde e seca, utilizaram parcelas de $12 \mathrm{~m}^{2}(4,8 \mathrm{~m} \times 2,5 \mathrm{~m})$, em diferentes espaçamentos e com quatro repetiçôes. Para a avaliaçáo de caracteres agronômicos, entre eles a massa verde, de três genótipos de milheto, Guimarães et al. (2009) usaram parcelas constituídas por quatro linhas com cinco metros de comprimento, espaçadas em $0,90 \mathrm{~m}$ e área útil de $18 \mathrm{~m}^{2}$, com quatro repetiçôes. Em experimento com semeadura a lanço, Marcante et al. (2011) utilizaram parcelas de $20 \mathrm{~m}^{2}$ $(5 \mathrm{~m} \times 4 \mathrm{~m}$ ) e cinco repetiçóes, para avaliar a fitomassa seca. Também em semeadura a lanço, Timossi et al. (2007) usaram parcelas de $300 \mathrm{~m}^{2}(6 \mathrm{~m} \times 50 \mathrm{~m})$ e oito repetiçôes, para a avaliaçáo do desenvolvimento de culturas de cobertura de solo, como capim-braquiária (Brachiaria decumbens), braquiarão (Brachiaria brizantha) e milheto (Pennisetum glaucum). Nos trabalhos supracitados, foi utilizado o delineamento blocos ao acaso, com exceção do trabalho realizado por Finholdt et al. (2009), no qual foi utilizado o delineamento inteiramente casualizado.

Estudos para a determinação do tamanho de parcela, avaliando a massa verde, têm sido realizados em plantas de cobertura de solo. Nas culturas de nabo-forrageiro (Raphanus sativus L.), de aveia-preta (Avena strigosa Schreb) e de feijão-de-porco (Canavalia ensiformis), o tamanho ótimo de parcela foi de 1,20 $\mathrm{m}^{2}$ (Cargnelutti et al., 2011), 4,14 $\mathrm{m}^{2}$ (Cargnelutti et al., 2014a) e 5,85 $\mathrm{m}^{2}$ (Cargnelutti et al., 2014b), respectivamente. No entanto, na cultura do milheto, estudos referentes ao tamanho de parcela e o número de repetiçôes para avaliar a massa verde de parte aérea náo foram encontrados na literatura. Assim, os objetivos deste trabalho foram determinar o tamanho ótimo de parcela e o número de repetiçóes, para avaliar a massa verde de parte aérea de milheto, em épocas de avaliação.

\section{MATERIAL E MÉTOdOS}

Foram conduzidos 42 ensaios de uniformidade com a cultura milheto (Pennisetum glaucum (L.) R. Brown), cv. comum, em área experimental de $28 \mathrm{~m} \times 60 \mathrm{~m}\left(1.680 \mathrm{~m}^{2}\right)$, localizada a $29^{\circ} 42^{\prime} \mathrm{S}, 53^{\circ} 49^{\prime} \mathrm{W}$ e a $95 \mathrm{~m}$ de altitude, no ano agrícola 2012-2013. A semeadura de milheto foi realizada a lanço, em 26 de outubro de 2012, na densidade de $20 \mathrm{~kg}$ ha $^{-1}$. A emergência ocorreu em 2 de novembro de 2012. A adubação de base foi de $40 \mathrm{~kg} \mathrm{ha}^{-1} \mathrm{de} \mathrm{N}, 160 \mathrm{~kg} \mathrm{ha}^{-1} \mathrm{de}$ $\mathrm{P}_{2} \mathrm{O}_{5}$ e $160 \mathrm{~kg} \mathrm{ha}^{-1}$ de $\mathrm{K}_{2} \mathrm{O}$, e em 12 de novembro de 2012 foram aplicados, em cobertura, $90 \mathrm{~kg}$ de $\mathrm{N} \mathrm{ha}^{-1}$.

Cada ensaio de uniformidade de tamanho $6 \mathrm{~m} \times 4 \mathrm{~m}$ $\left(24 \mathrm{~m}^{2}\right)$ foi dividido em 24 unidades experimentais básicas (UEB) de $1 \mathrm{~m} \times 1 \mathrm{~m}\left(1 \mathrm{~m}^{2}\right)$, resultando em uma matriz de seis linhas e quatro colunas. Foram avaliados 18 ensaios aos 52 dias após a semeadura (primeira época de avaliação), 6 ensaios aos 69 dias após a semeadura (segunda época de avaliação) e 18 ensaios aos 82 dias após a semeadura (terceira época de avaliação), totalizando 42 ensaios de uniformidade e 1.008 UEB de $1 \mathrm{~m}^{2}$. As avaliaçóes, em cada UEB de cada ensaio, consistiram no corte da parte aérea das plantas junto à superfície do solo e a sua pesagem para a obtenção da massa verde, em gramas $\mathrm{m}^{-2}$.

Conforme a metodologia de Paranaíba et al. (2009), para cada um dos 42 ensaios de uniformidade, com os dados de 
massa verde de parte aérea das $24 \mathrm{UEB}$, foram determinados o coeficiente de autocorrelaçáo espacial de primeira ordem $(\rho)$, a variância $\left(s^{2}\right)$, a média $(m)$ e o coeficiente de variação (CV, em \%) do ensaio. A estimativa de $\rho$ foi obtida no sentido das linhas. Para isso, iniciou-se o caminhamento na matriz a partir da UEB localizada na linha 1 , coluna 1 , até a linha 1 , coluna 4 , retornando a partir da linha 2 , coluna 4 , até a linha 2 , coluna 1, e assim sucessivamente, até concluir o caminhamento na UEB localizada na linha 6 , coluna 1 . Posteriormente para um dos 42 ensaios, foi calculado o tamanho ótimo de parcela $\left(\mathrm{Xo}, \mathrm{em} \mathrm{m}^{2}\right.$ ) pelo método da curvatura máxima do modelo do coeficiente de variação, pela expressáo

$X o=\frac{10 \sqrt[3]{2\left(1-\rho^{2}\right) s^{2} m}}{m}$

e determinado o coeficiente de variação no tamanho ótimo de parcela $\left(\mathrm{CV}_{\mathrm{Xo}}\right.$, em \%), pela expressão

$C V_{X o}=\frac{\sqrt{\left(1-\rho^{2}\right) s^{2} / m^{2}}}{\sqrt{X o}} \times 100$ (2) (Paranaíba et al., 2009).

Para as estimativas de $\rho, s^{2}, \mathrm{~m}, \mathrm{CV}$, Xo e $\mathrm{CV}_{\mathrm{Xo}}$, em cada época de avaliação, foram calculadas a média, o desvio-padrão, o coeficiente de variação e o valor-p do teste de normalidade de Kolmogorov-Smirnov. A comparaçáo de médias, das estatísticas $\rho, s^{2}, \mathrm{~m}, \mathrm{CV}$, Xo e $\mathrm{CV}_{\mathrm{Xo}}$, entre as épocas de avaliação, foi realizada pelo teste t de Student para amostras independentes, a 5\% de probabilidade de erro. Essas comparaçôes de médias foram realizadas duas a duas, e os resultados dessas comparaçóes foram representados por letras ao lado das médias.

A diferença mínima significativa (d) do teste de Tukey, em percentagem da média geral do experimento, é estimada pela expressão

$d=\frac{q_{\alpha(i ; G L E)} \sqrt{\frac{Q M E}{r}}}{m} \times 100$

sendo $q_{\alpha(i ; G L E)}$ o valor crítico do teste de Tukey em nível $\alpha$ de probabilidade de erro $(\alpha=0,05)$, i o número de tratamentos, GLE o número de graus de liberdade do erro, no caso, utiliza-se $\mathrm{i}(\mathrm{r}-1)$ para o delineamento inteiramente casualizado e (i-1)(r-1) para o delineamento blocos ao acaso, QME o quadrado médio do erro, r o número de repetiçôes e $\mathrm{m}$ a média do experimento. Substituindo a expressão do coeficiente de variação experimental

$\left(C V=\frac{\sqrt{Q M E}}{m} \times 100\right)$

em percentagem, na expressão de $\mathrm{d}$ e isolando $\mathrm{r}$, calcula-se o número de repetiçóes pela expressão

$r=\left(\frac{q_{\dot{a}(i ; G L E)} C V}{d}\right)^{2}$
O coeficiente de variação experimental da expressão corresponde ao $\mathrm{CV}_{\mathrm{Xo}_{\mathrm{o}}}$ e é expresso em percentagem, pois é o CV esperado para o experimento com Xo determinado (Cargnelutti et al., 2014a). Posteriormente, com base na média de $\mathrm{CV}_{\mathrm{Xo}}$, entre as três épocas de avaliação, determinou-se o número de repetições $(\mathrm{r})$ por processo iterativo até a convergência, para experimentos nos delineamentos inteiramente casualizados e blocos ao acaso, em cenários formados pelas combinaçóes de i $(\mathrm{i}=3,4, \ldots, 50)$ e d $(\mathrm{d}=10 \%, 12 \%, \ldots, 30 \%)$. As análises estatísticas foram realizadas com auxílio do aplicativo Microsoft Office Excel ${ }^{\circledR}$.

\section{RESULTADOS E DISCUSSÃO}

Foi constatada variabilidade das estatísticas: coeficiente de autocorrelação espacial de primeira ordem $(\rho)$, variância $\left(s^{2}\right)$, média $(\mathrm{m})$, coeficiente de variação do ensaio $(\mathrm{CV})$, tamanho ótimo de parcela (Xo) e coeficiente de variação no tamanho ótimo de parcela $\left(\mathrm{CV}_{\mathrm{Xo}_{0}}\right)$, obtidos a partir da massa verde de parte aérea de milheto, nos 18 ensaios da primeira época de avaliação $(12,99 \% \leq \mathrm{CV} \leq 170,61 \%)$, nos 6 ensaios da segunda época de avaliação $(6,50 \% \leq \mathrm{CV} \leq 521,66 \%)$ e nos 18 ensaios da terceira época de avaliação $(6,46 \% \leq \mathrm{CV} \leq 112,11 \%)$ (Tabela 1). Ampla variabilidade das estimativas entre os ensaios, em cada época de avaliação, também foi constatada em estudo de Cargnelutti et al. (2014a), ao utilizar o método da curvatura máxima do modelo do coeficiente de variação. Segundo os autores, esse cenário de variabilidade é importante em estudos de tamanho ótimo de parcela e de número de repetiçôes, pois expressa condiçóes reais da cultura a campo, ao longo do seu ciclo.

De acordo com a normalidade dos dados verificada por meio do teste de Kolmogorov-Smirnov, todas as estatísticas ( $\rho, s^{2}$, $\mathrm{m}, \mathrm{CV}, \mathrm{Xo} \mathrm{e} \mathrm{CV}_{\mathrm{Xo}_{\mathrm{o}}}$ ) apresentaram boa aderência à distribuiçáo normal na primeira $(\mathrm{p} \geq 0,115)$, na segunda $(\mathrm{p} \geq 0,524)$ e na terceira época de avaliação $(\mathrm{p} \geq 0,633)$ (Tabela 1$)$. Como os dados se ajustaram à distribuição normal, existe maior concentração de ensaios com os valores das estatísticas $\rho$, $s^{2}, \mathrm{~m}, \mathrm{CV}$, Xo e $\mathrm{CV}_{\mathrm{Xo}}$ próximas ao valor central e poucos ensaios com valores extremos (altos e baixos). Com isso, é possível utilizar as informaçóes centrais dessa distribuição. E como não houve diferença significativa entre as épocas de avaliação para as estatísticas $\rho, s^{2}, \mathrm{CV}$, Xo e $\mathrm{CV}_{\mathrm{Xo}}$, os valores médios de $\rho=0,11, s^{2}=1.613 .363,37, C V=21,90 \%$, $\mathrm{Xo}_{\mathrm{o}}=4,46 \mathrm{~m}^{2}$ e CV $\mathrm{X}_{\mathrm{o}}=9,96 \%$ são apropriados para representar as três épocas de avaliação.

A média de massa verde dos ensaios foi maior na terceira época de avaliação $\left(58,83 \mathrm{tha}^{-1}\right)$ e diferiu da segunda época de avaliaçấo $\left(55,25 \mathrm{t} \mathrm{ha}^{-1}\right)$, que obteve a menor média de massa verde. A média de massa verde da primeira época de avaliaçáo $\left(57,29 \mathrm{t} \mathrm{ha}^{-1}\right)$ não diferiu estatisticamente da segunda e da terceira época (Tabela 1). Os valores médios de massa verde foram superiores aos verificados 
Tabela 1. Coeficiente de autocorrelação espacial de primeira ordem $(\rho)$, variância $\left(s^{2}\right)$, média $(m)$, coeficiente de variação do ensaio $(C V$, em \%), tamanho ótimo de parcela $\left(\mathrm{Xo}_{0}, \mathrm{em} \mathrm{m}^{2}\right)$ e coeficiente de variação no tamanho ótimo de parcela $\left(\mathrm{CV}_{\mathrm{Xo}}\right.$, em \%), para massa verde de parte aérea (gramas $\mathrm{m}^{-2}$ ) de milheto (Pennisetum glaucum (L.) R. Brown), avaliada em 18 ensaios de uniformidade aos 52 dias após a semeadura (DAS) (primeira época de avaliação), 6 ensaios de uniformidade aos 69DAS (segunda época de avaliação) e 18 ensaios de uniformidade aos 82DAS (terceira época de avaliação), no ano agrícola 2012-2013

\begin{tabular}{|c|c|c|c|c|c|c|}
\hline Ensaio(1) & $\rho$ & $s^{2}$ & m & CV (\%) & Xo $\left(m^{2}\right)$ & $\mathrm{CV}_{\mathrm{xo}}(\%)$ \\
\hline & \multicolumn{6}{|c|}{ Primeira época de avaliação = 52 dias após a semeadura } \\
\hline 1 & 0,08 & $989.293,67$ & $5.805,75$ & 17,13 & 3,88 & 8,67 \\
\hline 2 & 0,04 & $1.239 .379,61$ & $5.786,29$ & 19,24 & 4,20 & 9,39 \\
\hline 3 & $-0,27$ & $1.102 .409,04$ & $6.290,08$ & 16,69 & 3,73 & 8,33 \\
\hline 4 & 0,44 & $2.227 .891,03$ & $4.917,38$ & 30,35 & 5,30 & 11,84 \\
\hline 5 & 0,72 & $2.646 .996,11$ & $3.576,25$ & 45,49 & 5,86 & 13,11 \\
\hline 6 & 0,01 & $744.076,42$ & $4.364,38$ & 19,76 & 4,27 & 9,56 \\
\hline 7 & 0,06 & $667.662,32$ & $6.289,17$ & 12,99 & 3,23 & 7,22 \\
\hline 8 & 0,19 & $2.424 .435,04$ & $6.363,46$ & 24,47 & 4,87 & 10,89 \\
\hline 9 & 0,29 & $1.423 .522,43$ & $5.915,21$ & 20,17 & 4,20 & 9,40 \\
\hline 10 & 0,06 & $3.294 .982,87$ & $5.879,46$ & 30,87 & 5,75 & 12,85 \\
\hline 11 & 0,08 & $1.326 .165,74$ & $5.821,50$ & 19,78 & 4,27 & 9,55 \\
\hline 12 & 0,32 & $1.442 .113,99$ & $5.928,58$ & 20,26 & 4,20 & 9,38 \\
\hline 13 & 0,15 & $2.249 .513,73$ & $6.314,58$ & 23,75 & 4,79 & 10,72 \\
\hline 14 & 0,05 & $2.172 .434,93$ & $6.338,33$ & 23,25 & 4,76 & 10,65 \\
\hline 15 & 0,16 & $677.067,43$ & $5.621,71$ & 14,64 & 3,47 & 7,76 \\
\hline 16 & $-0,18$ & $1.541 .693,39$ & $6.336,46$ & 19,60 & 4,20 & 9,40 \\
\hline 17 & $-0,09$ & $680.901,99$ & $5.736,42$ & 14,38 & 3,45 & 7,71 \\
\hline 18 & 0,30 & $1.075 .946,78$ & $5.840,92$ & 17,76 & 3,86 & 8,62 \\
\hline Média ${ }^{(2)}$ & $0,13 \mathrm{a}$ & $1.551 .471,47 \mathrm{a}$ & $5.729,22 a b$ & $21,70 \mathrm{a}$ & $4,35 \mathrm{a}$ & $9,72 \mathrm{a}$ \\
\hline Desvio padrão & 0,23 & $774.914,30$ & 743,94 & 7,68 & 0,75 & 1,68 \\
\hline CV (\%) & 170,61 & 49,95 & 12,99 & 35,39 & 17,27 & 17,27 \\
\hline \multirow[t]{2}{*}{ Valor-p ${ }^{(3)}$} & 0,804 & 0,663 & 0,115 & 0,246 & 0,429 & 0,429 \\
\hline & \multicolumn{6}{|c|}{ Segunda época de avaliação = 69 dias após a semeadura } \\
\hline 1 & 0,13 & $732.958,86$ & $5.261,42$ & 16,27 & 3,73 & 8,35 \\
\hline 2 & 0,11 & $928.753,12$ & $5.231,08$ & 18,42 & 4,06 & 9,09 \\
\hline 3 & $-0,02$ & $1.278 .886,78$ & $5.140,79$ & 22,00 & 4,59 & 10,27 \\
\hline 4 & $-0,30$ & $2.934 .114,43$ & $6.013,50$ & 28,48 & 5,29 & 11,82 \\
\hline 5 & 0,14 & $2.305 .742,41$ & $5.749,83$ & 26,41 & 5,15 & 11,52 \\
\hline 6 & 0,14 & $1.690 .536,22$ & $5.754,29$ & 22,60 & 4,64 & 10,38 \\
\hline Média $^{(2)}$ & $0,03 a$ & $1.645 .165,30 \mathrm{a}$ & $5.525,15 b$ & $22,36 \mathrm{a}$ & $4,58 \mathrm{a}$ & $10,24 \mathrm{a}$ \\
\hline Desvio padrão & 0,17 & $846.089,53$ & 359,23 & 4,62 & 0,60 & 1,35 \\
\hline CV (\%) & 521,66 & 51,43 & 6,50 & 20,65 & 13,16 & 13,16 \\
\hline \multirow[t]{2}{*}{ Valor-p ${ }^{(3)}$} & 0,524 & 0,996 & 0,780 & 0,999 & 0,993 & 0,993 \\
\hline & \multicolumn{6}{|c|}{ Terceira época de avaliação = 82 dias após a semeadura } \\
\hline 1 & 0,11 & $1.249 .659,04$ & $5.965,42$ & 18,74 & 4,11 & 9,19 \\
\hline 2 & $-0,07$ & $1.221 .312,17$ & $6.057,00$ & 18,25 & 4,05 & 9,05 \\
\hline 3 & 0,36 & $2.228 .808,14$ & $5.820,33$ & 25,65 & 4,86 & 10,86 \\
\hline 4 & $-0,06$ & $2.147 .487,59$ & $6.687,75$ & 21,91 & 4,57 & 10,23 \\
\hline 5 & 0,33 & $2.339 .375,98$ & $5.595,38$ & 27,34 & 5,10 & 11,41 \\
\hline 6 & 0,38 & $1.503 .167,78$ & $5.403,29$ & 22,69 & 4,45 & 9,95 \\
\hline 7 & $-0,05$ & $853.512,78$ & $6.210,00$ & 14,88 & 3,53 & 7,90 \\
\hline 8 & 0,05 & $2.390 .318,22$ & $5.758,96$ & 26,85 & 5,24 & 11,71 \\
\hline 9 & 0,04 & $1.001 .091,36$ & $6.378,83$ & 15,69 & 3,66 & 8,19 \\
\hline 10 & $-0,08$ & $2.373 .751,39$ & $6.272,00$ & 24,56 & 4,93 & 11,03 \\
\hline 11 & 0,37 & $2.048 .712,03$ & $5.804,88$ & 24,66 & 4,72 & 10,55 \\
\hline 12 & 0,15 & $1.398 .391,80$ & $5.415,67$ & 21,84 & 4,53 & 10,13 \\
\hline 13 & 0,21 & $916.958,82$ & $6.363,96$ & 15,05 & 3,51 & 7,85 \\
\hline 14 & 0,37 & $1.805 .834,37$ & $5.926,75$ & 22,67 & 4,46 & 9,98 \\
\hline 15 & $-0,05$ & $1.719 .724,02$ & $5.514,25$ & 23,78 & 4,83 & 10,81 \\
\hline
\end{tabular}

(1) Cada ensaio de uniformidade de tamanho $6 \mathrm{~m} \times 4 \mathrm{~m}\left(24 \mathrm{~m}^{2}\right)$ foi dividido em 24 unidades experimentais básicas de $1 \mathrm{~m} \times 1 \mathrm{~m}(1 \mathrm{~m})$, formando uma matriz de seis linhas e quatro colunas. ${ }^{(2)}$ Para cada estatística $\left(\rho, s^{2}, \mathrm{~m}, \mathrm{CV}, \mathrm{Xo} \mathrm{e} \mathrm{CV}_{\mathrm{Xo}_{\mathrm{o}}}\right)$ as médias nāo seguidas por mesma letra na coluna (comparação de médias entre as épocas de avaliaçáo) diferem a $5 \%$ de probabilidade pelo teste t de Student para amostras independentes. ${ }^{(3)}$ Teste de normalidade de Kolmogorov-Smirnov. 
Tabela 1. Continuaçấo...

\begin{tabular}{|c|c|c|c|c|c|c|}
\hline \multicolumn{7}{|c|}{ Terceira época de avaliação = 82 dias após a semeadura } \\
\hline 16 & 0,26 & $1.684 .552,95$ & $5.367,92$ & 24,18 & 4,78 & 10,69 \\
\hline 17 & 0,15 & $1.458 .743,17$ & $5.674,96$ & 21,28 & 4,46 & 9,97 \\
\hline 18 & 0,32 & $1.240 .758,70$ & $5.692,50$ & 19,57 & 4,10 & 9,16 \\
\hline Média(2) & $0,16 \mathrm{a}$ & $1.643 .453,35 \mathrm{a}$ & $5.883,88 \mathrm{a}$ & $21,64 \mathrm{a}$ & $4,44 \mathrm{a}$ & $9,93 \mathrm{a}$ \\
\hline Desvio padrão & 0,17 & $517.003,38$ & 380,37 & 3,88 & 0,52 & 1,16 \\
\hline CV (\%) & 112,11 & 31,46 & 6,46 & 17,92 & 11,64 & 11,64 \\
\hline Valor-p ${ }^{(3)}$ & 0,727 & 0,967 & 0,952 & 0,917 & 0,633 & 0,633 \\
\hline
\end{tabular}

(1) Cada ensaio de uniformidade de tamanho $6 \mathrm{~m} \times 4 \mathrm{~m}\left(24 \mathrm{~m}^{2}\right)$ foi dividido em 24 unidades experimentais básicas de $1 \mathrm{~m} \times 1 \mathrm{~m}(1 \mathrm{~m})$, formando uma matriz de seis linhas e quatro colunas. ${ }^{(2)}$ Para cada estatística $\left(\rho, s^{2}, \mathrm{~m}, \mathrm{CV}, \mathrm{Xo}\right.$ e $\left.\mathrm{CV}_{\mathrm{X}_{\mathrm{o}}}\right)$ as médias năo seguidas por mesma letra na coluna (comparação de médias entre as épocas de avaliação) diferem a $5 \%$ de probabilidade pelo teste t de Student para amostras independentes. ${ }^{(3)}$ Teste de normalidade de Kolmogorov-Smirnov.

por Guimarães et al. (2009), que avaliaram três genótipos de milheto, em épocas de avaliação, e obtiveram médias de $28,87 \mathrm{tha}^{-1}, 29,22 \mathrm{tha}^{-1}$ e $30,45 \mathrm{tha}^{-1}$, aos 52, 67 e 82 dias após a semeadura (DAS), respectivamente. Ainda, um dos genótipos (CMS-1) apresentou comportamento semelhante ao presente estudo, com maior produção de massa verde aos 82DAS e menor produção de massa verde aos 67DAS. Contudo, os autores verificaram que as épocas de avaliação não tiveram efeito significativo para os três genótipos.

A massa verde de parte aérea de milheto oscilou entre $3.576,25$ gramas $\mathrm{m}^{-2}$ no ensaio 5 da primeira época de avaliação e $6.687,75$ gramas $\mathrm{m}^{-2}$ no ensaio 4 da terceira época de avaliação. A média dos 42 ensaios nas três épocas de avaliaçáo foi de $5.712,75$ gramas $\mathrm{m}^{-2}$ ou $57,12 \mathrm{t} \mathrm{ha}^{-1}$ (Tabela 1). Em estudo desenvolvido por Finholdt et al. (2009), foram obtidas $58,10 \mathrm{t} \mathrm{ha}^{-1}$ de massa verde de milheto aos 50DAS. Dessa forma, a média de massa verde dos ensaios da primeira época de avaliação (52DAS) e a média geral foram semelhantes às obtidas por Finholdt et al. (2009), em experimento realizado em Uberaba, Estado de Minas Gerais. Em trabalho desenvolvido por Costa et al. (2013), também obtiveram-se valores semelhantes aos do presente estudo, com massa verde de 57,18 e 55,95 $\mathrm{t} \mathrm{ha}^{-1}$, em pleno florescimento, na densidade de semeadura de 25 e $50 \mathrm{~kg} \mathrm{ha}^{-1}$, respectivamente, utilizando uma cultivar comum. Valor inferior de massa verde de $44,16 \mathrm{t} \mathrm{ha}^{-1}$, foi obtido em estudo de Priesnitz et al. (2011) em uma cultivar comum, em estádio de maturação fisiológica. Valores inferiores também foram verificados por Oliveira et al. (2002) e Suzuki $\&$ Alves (2006). Em relação à primeira época de avaliação (52DAS), valor superior de massa verde de $63,52 \mathrm{t} \mathrm{ha}^{-1}$ foi obtido por Boer et al. (2008), aos 53DAS, utilizando a variedade ADR 500. Isso pode ser explicado pela utilização de uma variedade melhorada geneticamente para a produção de biomassa. Essas diferenças de massa verde constatadas podem estar relacionadas às variedades usadas, às condiçôes edafoclimáticas predominantes em cada local e aos períodos de avaliaçáo e tempo de manejo da cultura (Boer et al., 2008). Portanto, pode-se destacar que, em geral, a cultura teve desenvolvimento adequado e elevado potencial produtivo nas três épocas de avaliação, o que confere credibilidade aos resultados obtidos.

Deve ser considerado ainda que, dependendo da variedade, a produção das plantas de milheto tem desempenho distinto, principalmente se o manejo ocorrer em diferentes estádios fenológicos (Pires et al., 2007). Nesse sentido, Silva et al. (2002) também ressaltam que a época de corte de plantas de cobertura de solo, no caso, o milheto, deve ser de preferência após o florescimento e antes da frutificação, para garantir a adição de uma boa quantidade de material vegetal ao solo. Expressivas produçôes de massa verde promovem uma cobertura de solo eficiente, podendo até favorecer a supressão de plantas daninhas, reduzindo os custos para o seu controle (Pires et al., 2007) e ainda melhorando as condiçôes do solo para as culturas sucessoras. Por isso, a importância do estudo com a cultura do milheto em épocas de avaliaçáo para verificar a influência da variabilidade de produção de massa verde no tamanho de parcela, ao longo das épocas.

A média dos coeficientes de variação $(\mathrm{CV})$ de massa verde oscilou entre $21,64 \%$ na terceira época de avaliação e $22,36 \%$ na segunda época de avaliação (Tabela 1 ). Coeficientes de variação dessa magnitude estão acima do valor observado de CV de 10,62\%, em trabalho realizado por Guimaráes et al. (2009), mensurando a massa verde de parte aérea de três genótipos de milheto, em épocas de avaliação. E também estão acima do valor verificado em trabalho realizado por Priesnitz et al. (2011), que obtiveram CV de $17,67 \%$ e $16,40 \%$, avaliando a massa verde de duas cultivares de milheto, em estádio de grão pastoso e na maturação fisiológica, respectivamente. Esses valores são considerados aceitáveis pelo fato de que os experimentos conduzidos a campo geralmente apresentam estimativas de coeficientes de variação mais elevadas, devido ao menor controle do erro experimental (Smiderle et al., 2014).

Nem todos os tamanhos de parcela estimados são operacionalmente viáveis, devido ao custo para condução de experimentos, demanda de mão de obra, tempo e as limitaçôes da área experimental. Os tamanhos ótimos de parcela, estimados pelo método da curvatura máxima do modelo do coeficiente de variação, para avaliar a massa verde 
de parte aérea de milheto, não apresentaram diferenças entre as três épocas de avaliação. Esses resultados indicam que as mudanças ambientais ocorridas ao longo das épocas de avaliação não foram suficientes para alterar as estatísticas estudadas $\left(\rho, s^{2}, \mathrm{CV}\right.$, Xo e $\mathrm{CV}_{\mathrm{Xo}_{0}}$ ), com exceção da média, que apresentou diferenças significativas nas épocas de avaliação, porém praticamente estabilizou sua produção de massa verde aos 52DAS. Logo, o valor médio de tamanho ótimo de parcela para as três épocas de avaliação, no ano agrícola 2012-2013, foi de 4,46UEB de $1 \mathrm{~m}^{2}\left(4,46 \mathrm{~m}^{2}\right)$, e o coeficiente de variação no tamanho ótimo de parcela foi de 9,96\% (Tabela 1). Nos estudos de Timossi et al. (2007), Guimarães et al. (2009), Marcante et al. (2011) e Priesnitz et al. (2011) foram utilizadas parcelas de tamanho superior ao Xo do presente estudo, o que pode indicar que as informaçôes obtidas nesses estudos apresentam credibilidade.

Em cenários formados pelas combinaçôes de três tratamentos variando até 50 tratamentos e diferenças mínimas entre médias de tratamentos a serem detectadas como significativas a $5 \%$ de probabilidade, pelo teste de Tukey, expressas em percentagem da média do experimento $(\mathrm{d}=10 \%, 12 \%, \ldots, 30 \%)$, para avaliar a massa verde de parte aérea de milheto no delineamento inteiramente casualizado (DIC), o número de repetiçôes oscilou entre 2,63 (três tratamentos e $\mathrm{d}=30 \%$ ) e 31,76 (50 tratamentos e $\mathrm{d}=10 \%$ ) (Tabela 2), e no delineamento blocos ao acaso (DBA) variou de 2,35 (três tratamentos e $\mathrm{d}=30 \%$ ) a 31,76 (50 tratamentos e $\mathrm{d}=10 \%$ ) (Tabela 3 ).

Em um experimento com 50 tratamentos e maior precisão experimental $(\mathrm{d}=10 \%)$, são necessárias 31,76 repetiçōes, nos delineamentos DIC e DBA, o que torna inviável a realização de experimentos com esse elevado número de repetiçôes (Tabelas 2 e 3). Com as informaçóes disponíveis nas tabelas 2 e 3 e utilizando o tamanho de parcela de $4,46 \mathrm{~m}^{2}$, o pesquisador pode fazer a combinaçáo de tratamentos, diferenças mínimas entre médias de tratamentos e número de repetiçôes que julgar necessário, conforme a disponibilidade de área experimental, de tempo e de recursos humanos e financeiros.

Em um experimento utilizando DIC ou DBA e fixando o $\mathrm{Xo}$, o $\mathrm{CV}_{\mathrm{Xo}}$ e d, com o acréscimo do número de tratamentos, o número de repetiçôes aumenta (Tabelas 2 e 3), assim como foi comprovado por Cargnelutti et al. (2014a) e Cargnelutti et al. (2014b). E, conforme aumenta o número de tratamentos, o número de repetiçóes para DBA e para DIC se aproxima. Logo, para avaliar 50 tratamentos e d=30\%, são necessárias 3,69 repetições em DIC e DBA (Tabelas 2 e 3). Na prática, em experimentos a campo não é possível utilizar 3,69 repetiçôes, então, o correto é utilizar quatro repetiçôes. Esse número de repetições $(r=4)$, também foi usado em trabalhos realizados por Finholdt et al. (2009), Guimarães et al. (2009) e Priesnitz et al. (2011).

A dimensão adequada da parcela e o número de repetiçôes adequado conferem maior precisão experimental e qualificam a inferência dos resultados, pois a finalidade de um experimento é detectar a existência de diferenças significativas entre tratamentos testados (Donato et al., 2008). Com base na fórmula da diferença mínima significativa (d) do teste de Tukey, expressa em percentagem da média do experimento, utilizando i=50, $\alpha=0,05, \mathrm{CV}_{\mathrm{X}_{\mathrm{o}}}=9,96 \%$ e $\mathrm{r}=4$, obtém-se $\mathrm{d}=28,74 \%$, para o delineamento inteiramente casualizado e d=28,75\%, para o delineamento blocos ao acaso. Então, pode-se inferir que, para avaliar massa verde de parte aérea de milheto em experimentos nos delineamentos DIC e DBA com até 50 tratamentos, quatro repetiçóes são suficientes para identificar pelo teste de Tukey, a 5\% de probabilidade, diferenças significativas entre médias de tratamentos de $28,75 \%$ da média do experimento.

Tabela 2. Número de repetiçôes, para avaliar a massa verde de parte aérea de milheto (Pennisetum glaucum (L.) R. Brown), em experimentos no delineamento inteiramente casualizado, em cenários formados pelas combinaçóes de i tratamentos (i=3, 4, ..., 50) e d diferenças mínimas entre médias de tratamentos a serem detectadas como significativas a 5\% de probabilidade, pelo teste de Tukey, expressas em percentagem da média do experimento $(\mathrm{d}=10 \%, 12 \%, \ldots, 30 \%)$, a partir de tamanho ótimo de parcela $\left(\mathrm{Xo}=4,46 \mathrm{~m}^{2}\right)$ e coeficiente de variaçáo no tamanho ótimo de parcela $\left(\mathrm{CV}_{\mathrm{Xo}_{0}}=9,96 \%\right)$

\begin{tabular}{|c|c|c|c|c|c|c|c|c|c|c|c|}
\hline $\mathbf{i}$ & $10 \%$ & $12 \%$ & $14 \%$ & $16 \%$ & $18 \%$ & $20 \%$ & $22 \%$ & $24 \%$ & $26 \%$ & $28 \%$ & $30 \%$ \\
\hline 3 & 11,95 & 8,64 & 6,66 & 5,38 & 4,52 & 3,90 & 3,46 & 3,13 & 2,98 & 2,72 & 2,63 \\
\hline 4 & 13,98 & 9,99 & 7,60 & 6,06 & 5,01 & 4,26 & 3,72 & 3,30 & 2,99 & 2,77 & 2,65 \\
\hline 5 & 15,53 & 11,03 & 8,33 & 6,58 & 5,39 & 4,55 & 3,93 & 3,46 & 3,10 & 2,83 & 2,65 \\
\hline 6 & 16,79 & 11,88 & 8,92 & 7,01 & 5,71 & 4,78 & 4,11 & 3,60 & 3,21 & 2,91 & 2,67 \\
\hline 7 & 17,85 & 12,60 & 9,43 & 7,38 & 5,99 & 4,99 & 4,26 & 3,71 & 3,29 & 2,96 & 2,70 \\
\hline 8 & 18,78 & 13,22 & 9,87 & 7,71 & 6,23 & 5,17 & 4,40 & 3,82 & 3,37 & 3,02 & 2,74 \\
\hline 9 & 19,60 & 13,77 & 10,27 & 8,00 & 6,44 & 5,34 & 4,53 & 3,92 & 3,44 & 3,07 & 2,79 \\
\hline 10 & 20,33 & 14,27 & 10,62 & 8,26 & 6,64 & 5,49 & 4,64 & 4,01 & 3,51 & 3,13 & 2,82 \\
\hline 11 & 20,99 & 14,72 & 10,94 & 8,49 & 6,82 & 5,63 & 4,75 & 4,09 & 3,58 & 3,18 & 2,86 \\
\hline 12 & 21,60 & 15,13 & 11,24 & 8,71 & 6,99 & 5,76 & 4,85 & 4,17 & 3,64 & 3,22 & 2,90 \\
\hline 13 & 22,16 & 15,52 & 11,51 & 8,92 & 7,14 & 5,88 & 4,94 & 4,24 & 3,70 & 3,27 & 2,93 \\
\hline 14 & 22,68 & 15,87 & 11,77 & 9,11 & 7,29 & 5,99 & 5,03 & 4,31 & 3,75 & 3,31 & 2,96 \\
\hline 15 & 23,17 & 16,20 & 12,00 & 9,28 & 7,42 & 6,09 & 5,11 & 4,37 & 3,80 & 3,35 & 2,99 \\
\hline
\end{tabular}


Tabela 2. Continuação...

\begin{tabular}{|c|c|c|c|c|c|c|c|c|c|c|c|}
\hline i & $10 \%$ & $12 \%$ & $14 \%$ & $16 \%$ & $18 \%$ & $20 \%$ & $22 \%$ & $24 \%$ & $26 \%$ & $28 \%$ & $30 \%$ \\
\hline 16 & 23,62 & 16,51 & 12,23 & 9,45 & 7,55 & 6,19 & 5,19 & 4,44 & 3,85 & 3,39 & 3,02 \\
\hline 17 & 24,05 & 16,80 & 12,44 & 9,61 & 7,67 & 6,29 & 5,27 & 4,49 & 3,90 & 3,43 & 3,05 \\
\hline 18 & 24,45 & 17,08 & 12,64 & 9,75 & 7,78 & 6,37 & 5,34 & 4,55 & 3,94 & 3,46 & 3,08 \\
\hline 19 & 24,83 & 17,34 & 12,82 & 9,90 & 7,89 & 6,46 & 5,40 & 4,60 & 3,99 & 3,50 & 3,11 \\
\hline 20 & 25,20 & 17,59 & 13,00 & 10,03 & 7,99 & 6,54 & 5,47 & 4,66 & 4,03 & 3,53 & 3,14 \\
\hline 21 & 25,54 & 17,83 & 13,17 & 10,16 & 8,09 & 6,62 & 5,53 & 4,71 & 4,07 & 3,56 & 3,16 \\
\hline 22 & 25,87 & 18,05 & 13,34 & 10,28 & 8,19 & 6,69 & 5,59 & 4,75 & 4,11 & 3,60 & 3,19 \\
\hline 23 & 26,19 & 18,27 & 13,49 & 10,40 & 8,28 & 6,76 & 5,65 & 4,80 & 4,14 & 3,63 & 3,21 \\
\hline 24 & 26,49 & 18,48 & 13,64 & 10,51 & 8,36 & 6,83 & 5,70 & 4,84 & 4,18 & 3,65 & 3,24 \\
\hline 25 & 26,78 & 18,68 & 13,79 & 10,62 & 8,45 & 6,90 & 5,75 & 4,89 & 4,21 & 3,68 & 3,26 \\
\hline 26 & 27,06 & 18,87 & 13,93 & 10,72 & 8,53 & 6,96 & 5,80 & 4,93 & 4,25 & 3,71 & 3,28 \\
\hline 27 & 27,33 & 19,05 & 14,06 & 10,82 & 8,61 & 7,02 & 5,85 & 4,97 & 4,28 & 3,74 & 3,30 \\
\hline 28 & 27,59 & 19,23 & 14,19 & 10,92 & 8,68 & 7,08 & 5,90 & 5,01 & 4,31 & 3,76 & 3,32 \\
\hline 29 & 27,84 & 19,40 & 14,32 & 11,02 & 8,75 & 7,14 & 5,95 & 5,04 & 4,34 & 3,79 & 3,34 \\
\hline 30 & 28,08 & 19,57 & 14,44 & 11,11 & 8,83 & 7,20 & 5,99 & 5,08 & 4,37 & 3,81 & 3,36 \\
\hline 31 & 28,32 & 19,73 & 14,55 & 11,19 & 8,89 & 7,25 & 6,04 & 5,12 & 4,40 & 3,84 & 3,38 \\
\hline 32 & 28,55 & 19,89 & 14,67 & 11,28 & 8,96 & 7,30 & 6,08 & 5,15 & 4,43 & 3,86 & 3,40 \\
\hline 33 & 28,77 & 20,04 & 14,78 & 11,36 & 9,02 & 7,35 & 6,12 & 5,18 & 4,46 & 3,88 & 3,42 \\
\hline 34 & 28,98 & 20,19 & 14,88 & 11,44 & 9,09 & 7,40 & 6,16 & 5,22 & 4,48 & 3,91 & 3,44 \\
\hline 35 & 29,19 & 20,33 & 14,99 & 11,52 & 9,15 & 7,45 & 6,20 & 5,25 & 4,51 & 3,93 & 3,46 \\
\hline 36 & 29,39 & 20,47 & 15,09 & 11,60 & 9,21 & 7,50 & 6,24 & 5,28 & 4,54 & 3,95 & 3,48 \\
\hline 37 & 29,59 & 20,60 & 15,19 & 11,67 & 9,27 & 7,54 & 6,27 & 5,31 & 4,56 & 3,97 & 3,49 \\
\hline 38 & 29,78 & 20,74 & 15,28 & 11,75 & 9,32 & 7,59 & 6,31 & 5,34 & 4,58 & 3,99 & 3,51 \\
\hline 39 & 29,97 & 20,86 & 15,38 & 11,82 & 9,38 & 7,63 & 6,35 & 5,37 & 4,61 & 4,01 & 3,53 \\
\hline 40 & 30,15 & 20,99 & 15,47 & 11,88 & 9,43 & 7,68 & 6,38 & 5,40 & 4,63 & 4,03 & 3,54 \\
\hline 41 & 30,33 & 21,11 & 15,56 & 11,95 & 9,48 & 7,72 & 6,41 & 5,42 & 4,65 & 4,05 & 3,56 \\
\hline 42 & 30,50 & 21,23 & 15,64 & 12,02 & 9,53 & 7,76 & 6,45 & 5,45 & 4,68 & 4,07 & 3,57 \\
\hline 43 & 30,67 & 21,35 & 15,73 & 12,08 & 9,58 & 7,80 & 6,48 & 5,48 & 4,70 & 4,08 & 3,59 \\
\hline 44 & 30,84 & 21,46 & 15,81 & 12,15 & 9,63 & 7,84 & 6,51 & 5,50 & 4,72 & 4,10 & 3,60 \\
\hline 45 & 31,00 & 21,57 & 15,89 & 12,21 & 9,68 & 7,88 & 6,54 & 5,53 & 4,74 & 4,12 & 3,62 \\
\hline 46 & 31,16 & 21,68 & 15,97 & 12,27 & 9,73 & 7,91 & 6,57 & 5,55 & 4,76 & 4,14 & 3,63 \\
\hline 47 & 31,31 & 21,79 & 16,05 & 12,33 & 9,77 & 7,95 & 6,60 & 5,58 & 4,78 & 4,15 & 3,65 \\
\hline 48 & 31,46 & 21,90 & 16,13 & 12,38 & 9,82 & 7,99 & 6,63 & 5,60 & 4,80 & 4,17 & 3,66 \\
\hline 49 & 31,61 & 22,00 & 16,20 & 12,44 & 9,86 & 8,02 & 6,66 & 5,63 & 4,82 & 4,19 & 3,68 \\
\hline 50 & 31,76 & 22,10 & 16,28 & 12,50 & 9,91 & 8,06 & 6,69 & 5,65 & 4,84 & 4,20 & 3,69 \\
\hline
\end{tabular}

Tabela 3. Número de repetiçóes, para avaliar a massa verde de parte aérea de milheto (Pennisetum glaucum (L.) R. Brown), em experimentos no delineamento blocos ao acaso, em cenários formados pelas combinações de i tratamentos (i=3, 4, ..., 50) e d diferenças mínimas entre médias de tratamentos a serem detectadas como significativas a 5\% de probabilidade, pelo teste de Tukey, expressas em percentagem da média do experimento $(\mathrm{d}=10 \%, 12 \%, \ldots, 30 \%)$, a partir de tamanho ótimo de parcela $\left(\mathrm{Xo}=4,46 \mathrm{~m}^{2}\right)$ e coeficiente de variação no tamanho ótimo de parcela $\left(\mathrm{CV}_{\mathrm{Xo}_{0}}=9,96 \%\right)$

\begin{tabular}{|c|c|c|c|c|c|c|c|c|c|c|c|}
\hline i & $10 \%$ & $12 \%$ & $14 \%$ & $16 \%$ & $18 \%$ & $20 \%$ & $22 \%$ & $24 \%$ & $26 \%$ & $28 \%$ & $30 \%$ \\
\hline 3 & 12,44 & 9,14 & 7,14 & 5,86 & 5,00 & 4,36 & 3,79 & 3,21 & 2,51 & 2,41 & 2,35 \\
\hline 4 & 14,26 & 10,28 & 7,88 & 6,34 & 5,29 & 4,53 & 3,99 & 3,57 & 3,24 & 3,00 & 2,77 \\
\hline 5 & 15,71 & 11,22 & 8,51 & 6,77 & 5,57 & 4,73 & 4,11 & 3,64 & 3,27 & 3,00 & 2,80 \\
\hline 6 & 16,92 & 12,01 & 9,06 & 7,15 & 5,84 & 4,92 & 4,24 & 3,73 & 3,34 & 3,02 & 2,78 \\
\hline 7 & 17,95 & 12,70 & 9,53 & 7,48 & 6,09 & 5,09 & 4,37 & 3,81 & 3,39 & 3,07 & 2,81 \\
\hline 8 & 18,86 & 13,30 & 9,95 & 7,79 & 6,31 & 5,26 & 4,48 & 3,90 & 3,45 & 3,10 & 2,82 \\
\hline 9 & 19,66 & 13,84 & 10,33 & 8,06 & 6,51 & 5,41 & 4,59 & 3,98 & 3,51 & 3,14 & 2,85 \\
\hline 10 & 20,38 & 14,32 & 10,67 & 8,31 & 6,69 & 5,54 & 4,70 & 4,06 & 3,57 & 3,18 & 2,88 \\
\hline 11 & 21,04 & 14,76 & 10,99 & 8,54 & 6,87 & 5,67 & 4,80 & 4,14 & 3,63 & 3,22 & 2,91 \\
\hline 12 & 21,64 & 15,17 & 11,28 & 8,75 & 7,03 & 5,80 & 4,89 & 4,21 & 3,68 & 3,26 & 2,94 \\
\hline 13 & 22,19 & 15,55 & 11,54 & 8,95 & 7,17 & 5,91 & 4,98 & 4,27 & 3,73 & 3,30 & 2,97 \\
\hline 14 & 22,71 & 15,90 & 11,79 & 9,13 & 7,32 & 6,02 & 5,06 & 4,34 & 3,78 & 3,34 & 2,99 \\
\hline 15 & 23,19 & 16,23 & 12,03 & 9,31 & 7,45 & 6,12 & 5,14 & 4,40 & 3,83 & 3,38 & 3,02 \\
\hline
\end{tabular}


Tabela 3. Continuação...

\begin{tabular}{|c|c|c|c|c|c|c|c|c|c|c|c|}
\hline $\mathbf{i}$ & $10 \%$ & $12 \%$ & $14 \%$ & $16 \%$ & $18 \%$ & $20 \%$ & $22 \%$ & $24 \%$ & $26 \%$ & $28 \%$ & $30 \%$ \\
\hline 16 & 23,64 & 16,53 & 12,25 & 9,47 & 7,57 & 6,22 & 5,22 & 4,46 & 3,88 & 3,42 & 3,05 \\
\hline 17 & 24,07 & 16,82 & 12,46 & 9,63 & 7,69 & 6,31 & 5,29 & 4,52 & 3,92 & 3,45 & 3,08 \\
\hline 18 & 24,47 & 17,10 & 12,65 & 9,77 & 7,80 & 6,39 & 5,36 & 4,57 & 3,96 & 3,48 & 3,10 \\
\hline 19 & 24,85 & 17,36 & 12,84 & 9,91 & 7,91 & 6,48 & 5,42 & 4,62 & 4,00 & 3,52 & 3,13 \\
\hline 20 & 25,21 & 17,60 & 13,02 & 10,04 & 8,01 & 6,56 & 5,48 & 4,67 & 4,04 & 3,55 & 3,15 \\
\hline 21 & 25,56 & 17,84 & 13,19 & 10,17 & 8,11 & 6,63 & 5,54 & 4,72 & 4,08 & 3,58 & 3,18 \\
\hline 22 & 25,89 & 18,06 & 13,35 & 10,29 & 8,20 & 6,71 & 5,60 & 4,77 & 4,12 & 3,61 & 3,20 \\
\hline 23 & 26,20 & 18,28 & 13,51 & 10,41 & 8,29 & 6,78 & 5,66 & 4,81 & 4,16 & 3,64 & 3,23 \\
\hline 24 & 26,50 & 18,49 & 13,66 & 10,52 & 8,38 & 6,84 & 5,71 & 4,86 & 4,19 & 3,67 & 3,25 \\
\hline 25 & 26,79 & 18,69 & 13,80 & 10,63 & 8,46 & 6,91 & 5,76 & 4,90 & 4,22 & 3,69 & 3,27 \\
\hline 26 & 27,07 & 18,88 & 13,94 & 10,73 & 8,54 & 6,97 & 5,81 & 4,94 & 4,26 & 3,72 & 3,29 \\
\hline 27 & 27,34 & 19,06 & 14,07 & 10,83 & 8,62 & 7,03 & 5,86 & 4,98 & 4,29 & 3,75 & 3,31 \\
\hline 28 & 27,60 & 19,24 & 14,20 & 10,93 & 8,69 & 7,09 & 5,91 & 5,01 & 4,32 & 3,77 & 3,33 \\
\hline 29 & 27,85 & 19,41 & 14,32 & 11,02 & 8,76 & 7,15 & 5,96 & 5,05 & 4,35 & 3,80 & 3,35 \\
\hline 30 & 28,09 & 19,58 & 14,44 & 11,11 & 8,83 & 7,20 & 6,00 & 5,09 & 4,38 & 3,82 & 3,37 \\
\hline 31 & 28,33 & 19,74 & 14,56 & 11,20 & 8,90 & 7,26 & 6,04 & 5,12 & 4,41 & 3,84 & 3,39 \\
\hline 32 & 28,55 & 19,89 & 14,67 & 11,29 & 8,97 & 7,31 & 6,09 & 5,16 & 4,44 & 3,87 & 3,41 \\
\hline 33 & 28,77 & 20,04 & 14,78 & 11,37 & 9,03 & 7,36 & 6,13 & 5,19 & 4,46 & 3,89 & 3,43 \\
\hline 34 & 28,99 & 20,19 & 14,89 & 11,45 & 9,09 & 7,41 & 6,17 & 5,22 & 4,49 & 3,91 & 3,45 \\
\hline 35 & 29,20 & 20,33 & 14,99 & 11,53 & 9,15 & 7,46 & 6,20 & 5,25 & 4,52 & 3,93 & 3,47 \\
\hline 36 & 29,40 & 20,47 & 15,09 & 11,60 & 9,21 & 7,50 & 6,24 & 5,28 & 4,54 & 3,95 & 3,48 \\
\hline 37 & 29,59 & 20,61 & 15,19 & 11,68 & 9,27 & 7,55 & 6,28 & 5,31 & 4,57 & 3,97 & 3,50 \\
\hline 38 & 29,79 & 20,74 & 15,29 & 11,75 & 9,33 & 7,59 & 6,32 & 5,34 & 4,59 & 3,99 & 3,52 \\
\hline 39 & 29,97 & 20,87 & 15,38 & 11,82 & 9,38 & 7,64 & 6,35 & 5,37 & 4,61 & 4,01 & 3,53 \\
\hline 40 & 30,15 & 20,99 & 15,47 & 11,89 & 9,43 & 7,68 & 6,38 & 5,40 & 4,64 & 4,03 & 3,55 \\
\hline 41 & 30,33 & 21,12 & 15,56 & 11,96 & 9,49 & 7,72 & 6,42 & 5,43 & 4,66 & 4,05 & 3,56 \\
\hline 42 & 30,51 & 21,24 & 15,65 & 12,02 & 9,54 & 7,76 & 6,45 & 5,45 & 4,68 & 4,07 & 3,58 \\
\hline 43 & 30,67 & 21,35 & 15,73 & 12,09 & 9,59 & 7,80 & 6,48 & 5,48 & 4,70 & 4,09 & 3,59 \\
\hline 44 & 30,84 & 21,47 & 15,82 & 12,15 & 9,64 & 7,84 & 6,51 & 5,51 & 4,72 & 4,11 & 3,61 \\
\hline 45 & 31,00 & 21,58 & 15,90 & 12,21 & 9,68 & 7,88 & 6,55 & 5,53 & 4,75 & 4,12 & 3,62 \\
\hline 46 & 31,16 & 21,69 & 15,98 & 12,27 & 9,73 & 7,92 & 6,58 & 5,56 & 4,77 & 4,14 & 3,64 \\
\hline 47 & 31,32 & 21,79 & 16,05 & 12,33 & 9,78 & 7,95 & 6,60 & 5,58 & 4,79 & 4,16 & 3,65 \\
\hline 48 & 31,47 & 21,90 & 16,13 & 12,39 & 9,82 & 7,99 & 6,63 & 5,61 & 4,81 & 4,17 & 3,67 \\
\hline 49 & 31,62 & 22,00 & 16,20 & 12,44 & 9,87 & 8,02 & 6,66 & 5,63 & 4,83 & 4,19 & 3,68 \\
\hline 50 & 31,76 & 22,10 & 16,28 & 12,50 & 9,91 & 8,06 & 6,69 & 5,65 & 4,84 & 4,21 & 3,69 \\
\hline
\end{tabular}

\section{CONCLUSÃO}

O tamanho ótimo de parcela para avaliar a massa verde de parte aérea de milheto é de 4,46 unidades experimentais básicas de $1 \mathrm{~m}^{2}\left(4,46 \mathrm{~m}^{2}\right)$, para as três épocas de avaliação. Para avaliar até 50 tratamentos, nos delineamentos inteiramente casualizados e blocos ao acaso, quatro repetições são suficientes para que diferenças entre médias de tratamentos de $28,75 \%$ da média do experimento sejam significativas, pelo teste de Tukey, a 5\% de probabilidade.

\section{AGRADECIMENTOS}

Ao Conselho Nacional de Desenvolvimento Científico e Tecnológico (CNPq), à Coordenação de Aperfeiçoamento de Pessoal de Nível Superior (CAPES) e à Fundação de Amparo à Pesquisa do Estado do Rio Grande do Sul (FAPERGS), pela concessão de bolsas aos autores. Aos alunos bolsistas e voluntários, pelo auxílio na coleta de dados.

\section{REFERÊNCIAS}

Boer, C. A., Assis, R. L., Silva, G. P., Braz, A. J. B. P., Barroso, A. L. L., Cargnelutti, A., Fo., \& Pires, F. R. (2008). Biomassa, decomposição e cobertura do solo ocasionada por resíduos culturais de três espécies vegetais na regiáo centro-oeste do Brasil. Revista Brasileira de Ciência do Solo, 32, 843-851. http://dx.doi.org/10.1590/S010006832008000200038 .

Buso, W. H. D., França, A. F. S., \& Miyagi, E. S. (2014). Bromatological composition and dry matter digestibility of millet cultivars subjected to nitrogen doses. Arquivo Brasileiro de Medicina Veterinária e Zootecnia, 66, 887-893. http://dx.doi.org/10.1590/1678-41626746. 
Cargnelutti, A., Fo., Toebe, M., Burin, C., Fick, A. L., \& Casarotto, G. (2011). Tamanhos de parcela e de ensaio de uniformidade em nabo forrageiro. Ciência Rural, 41, 1517-1525. http://dx.doi.org/10.1590/ S0103-84782011005000119.

Cargnelutti, A., Fo., Alves, B. M., Toebe, M., Burin, C., Santos, G. O., Facco, G., Neu, I. M. M., \& Stefanello, R. B. (2014a). Tamanho de parcela e número de repetiçóes em aveia preta. Ciência Rural, 44, 1732-1739. http://dx.doi.org/10.1590/0103-8478cr20131466.

Cargnelutti, A., Fo., Toebe, M., Burin, C., Alves, B. M., Neu, I. M. M., Casarotto, G., \& Facco, G. (2014b). Tamanho de parcela e número de repetiçóes em feijão de porco. Ciência Rural, 44, 2142 2150. http://dx.doi.org/10.1590/0103-8478cr20140317.

Cazetta, D. A., Fornasieri, D. Fo., \& Girotto, F. (2005). Composição, produção de matéria seca e cobertura do solo em cultivo exclusivo e consorciado de milheto e crotalária. Acta Scientiarum. Agronomy, 27, 575-580. http://dx.doi.org/10.4025/actasciagron.v27i4.1298.

Costa, A. C. T., Erig, M. C., Reginatto, J. R., Domukoski, J. F., Ecco, M., Duarte, J. B. Jr., \& Vasconcelos, E. S. (2013). Comportamento do milheto na safra de verão em função do espaçamento entrelinhas e densidade de semeadura. Scientia Agraria Paranaensis, 12, 347-353. Recuperado em 27 de dezembro de 2014, de http://e-revista.unioeste. $\mathrm{br} /$ index.php/scientiaagraria/article/view/9556.

Donato, S. L. R., Siqueira, D. L., Silva, S. O., Cecon, P. R., Silva, J. A., \& Salomão, L. C. C. (2008). Estimativas de tamanho de parcelas para avaliaçáo de descritores fenotípicos em bananeira. Pesquisa Agropecuária Brasileira, 43, 957-969. http://dx.doi.org/10.1590/ S0100-204X2008000800003.

Finholdt, R. S., Assis, A. M., Bisinotto, F. F., \& Aquino, V. M. Jr., \& Silva, L. O. (2009). Avaliaçáo da biomassa e cobertura do solo de adubos verdes. FAZU em Revista, p.11-14. Recuperado em 27 de dezembro de 2014, de http://www.fazu.br/ojs/index.php/fazuemrevista/ article/view/36/30.

Guimarães, R. Jr., Gonçalves, L. C., Rodrigues, J. A. S., Pires, D. A. A., Jayme, D. G., Rodriguez, N. M., \& Saliba, E. O. S. (2009). Avaliação agronômica de genótipos de milheto (P. glaucum) plantados em período de safrinha. Archivos de Zootecnia, 58, 629-632. Recuperado em 27 de dezembro de 2014, de http://www.uco.es/organiza/servicios/ publica/az/php/img/web/19_14_17_37NotaAvaliacaoGuimaraes.pdf.

Marcante, N. C., Camacho, M. A., \& Paredes, F. P. J. (2011). Teores de nutrientes no milheto como cobertura de solo. Bioscience Journal, 27, 196-204. Recuperado em 27 de dezembro de 2014, de http:// www.seer.ufu.br/index.php/biosciencejournal/article/view/7564/7224.
Oliveira, T. K., Carvalho, G. J., \& Moraes, R. N. S. (2002). Plantas de cobertura e seus efeitos sobre o feijoeiro em plantio direto. Pesquisa Agropecuária Brasileira, 37, 1079-1087. http://dx.doi.org/10.1590/ S0100-204X2002000800005.

Paranaíba, P. F., Ferreira, D. F., \& Morais, A. R. (2009). Tamanho ótimo de parcelas experimentais: proposição de métodos de estimação. Revista Brasileira de Biometria, 27, 255-268. Recuperado em 27 de dezembro de 2014, de http://jaguar.fcav.unesp.br/RME/fasciculos/ v27/v27_n2/Patricia.pdf.

Pires, F. R., Assis, R. L., Silva, G. P., Braz, A. J. B. P., Santos, S. C., Vieira, S. A., No., \& Sousa, J. P. G. (2007). Desempenho agronômico de variedades de milheto em razão da fenologia em pré-safra. Bioscience Journal, 23, 41-49. Recuperado em 27 de dezembro de 2014, de http://www.seer.ufu.br/index.php/biosciencejournal/article/view/6695.

Priesnitz, R., Costa, A. C. T., Jandrey, P. E., Fréz, J. R. S., Duarte, J. B. Jr., \& Oliveira, P. S. R. (2011). Espaçamento entre linhas na produtividade de biomassa e de grãos em genótipos de milheto pérola. Semina: Ciências Agrárias, 32, 485-494. Recuperado em 27 de dezembro de 2014, de http://www.uel.br/revistas/uel/index.php/ semagrarias/article/viewFile/3394/8408.

Ramalho, M. A. P., Ferreira, D. F., \& Oliveira, A. C. (2012). Experimentação em genética e melhoramento de plantas. Lavras: UFLA. 305 p.

Silva, J. A. A., Vitti, G. C., Stuchi, E. S., \& Sempionato, O. R. (2002). Reciclagem e incorporaçáo de nutrientes ao solo pelo cultivo intercalar de adubos verdes em pomar de laranja-pêra. Revista Brasileira de Fruticultura, 24, 225-230. http://dx.doi.org/10.1590/ S0100-29452002000100048.

Smiderle, E. C., Botelho, F. B. S., Guilherme, S. R., Arantes, S. A. C. M., Botelho, R. T. C., \& Arantes, K. R. (2014). Tamanho de parcelas experimentais para a seleção de genótipos na cultura do feijoeiro. Comunicata Scientiae, 5, 51-58. Recuperado em 27 de dezembro de 2014, de http://comunicata.ufpi.br/index.php/comunicata/ article/view/218.

Storck, L., Garcia, D. C., Lopes, S. J., \& Estefanel, V. (2011). Experimentação vegetal. (3 ed.). Santa Maria: UFSM. 200 p.

Suzuki, L. E. A. S., \& Alves, M. C. (2006). Fitomassa de plantas de cobertura em diferentes sucessôes de culturas e sistemas de cultivo. Bragantia, 65, 121-127. http://dx.doi.org/10.1590/S000687052006000100016 .

Timossi, P. C., Durigan, J. C., \& Leite, G. J. (2007). Formação de palhada por braquiárias para adoção do sistema plantio direto. Bragantia, 66, 617-622. http://dx.doi.org/10.1590/S0006-87052007000400012. 